\title{
ON THE VARIABILITY OF THE NUCLEI OF THREE PLANETARY NEBULAE
}

\author{
M. A. KAZARIAN \\ (Burakan Astrophysical Observatory, U.S.S.R.)
}

The observations of nuclei of three planetary nebulae, NGC 6826, NGC 7662 and IC 4593, have been made as part of a more extensive program with the aim of obtaining their absolute spectrophotometric gradients $(\phi)$ and photographic magnitudes (from the spectrogram). The spectra were obtained with an 8-12" Schmidt telescope combined with an objective prism (the dispersion is $420 \AA / \mathrm{mm}$ at $\mathrm{H} \gamma$ ). The preliminary data which were obtained at different times, particularly the spectrophotometric gradients of the nuclei of these nebulae, did not agree with each other.

In order to find the causes of this disagreement we have made a rather long series of observations of the nucleus of NGC 6826. Relatively shorter observational series have also been made of the nuclei of NGC 7662 and of IC 4593. The spectral interval of the investigation was $\lambda \lambda 4100-4700$.

Twenty-two spectra of the nucleus of the NGC 6826 have been obtained on 15 different nights in the period from December, 1965 to October, 1966. The results show that the gradient changed within the limits 0.32 to $1 \cdot 46$, and the photographic magnitude between $9 .{ }^{\mathrm{m}} 6$ and $10^{\mathrm{m}} 3$.

Eight spectra of the nucleus of NGC 7662 were obtained on eight different nights in the period from August to December, 1966. The results showed that the gradient changed within the limits 0.50 to $1 \cdot 24$. No changes of the photographic magnitude were noticed in the period of our observations.

Six spectra of the nucleus of the IC 4593 were obtained on four different nights in the period from March to August, 1966. The results show that the gradient remained almost unchanged, but the photographic magnitude changed within the limits $10 \mathrm{~m} 2$ to $11^{\mathrm{m}} 1$.

Table 1

Summary of changes of nuclei of planetary nebulae

\begin{tabular}{|c|c|c|c|c|}
\hline Nebula & $n$ & $\begin{array}{c}\text { Period of } \\
\text { observation }\end{array}$ & Range of $\phi$ & Range of $m_{\mathrm{pg}}$ \\
\hline NGC 6826 & 22 & Dec., 1965 - Oct., 1966 & $0 \cdot 32-1.42$ & $9: 6-10^{m} \cdot 3$ \\
\hline NGC 7662 & 8 & August-Dec., 1966 & $0.50-1.24$ & no changes \\
\hline IC 4593 & 6 & March-Aug., 1966 & no changes & $10 \cdot 2-11 \cdot 1$ \\
\hline
\end{tabular}

Osterbrock and O'Dell (eds.), Planetary Nebulae, 381-382. I.A.U. 
The quantity of the observational material for the nuclei of NGC 7662 and IC 4593 is not sufficient to make it possible to be certain about the variation of these two nuclei. Therefore we may suppose that the nuclei of these nebulae are possibly variable objects, while the nucleus of NGC 6826 is definitely variable. 